\title{
Uso de sensores inerciales en fisioterapia: Una aproximación a procesos de evaluación del movimiento humano
}

\author{
Use of inertial sensors in physiotherapy: an approach to human movement assessment processes \\ Julialba Castellanos-Rúzi,2 orcid.org/0000-0003-3525-4484 \\ Lina María Montealegre-Mesa2* orcid.org/0000-0001-8176-4608 \\ Brahian Daniel Martínez-Toro1,3 orcid.org/0000-0002-4857-7330 \\ Juan José Gallo-Serna1,3 orcid.org/0000-0002-6186-1862 \\ Osvaldo Almanza Fuentes ${ }^{4}$ orcid.org/0000-0002-9643-0691
}

1. Programa Fisioterapia, Universidad Autónoma de Manizales. Manizales, Colombia.

2. Grupo Cuerpo Movimiento, Universidad Autónoma de Manizales. Manizales, Colombia.

3. Semillero de investigación TAMIF. Universidad Autónoma de Manizales. Manizales, Colombia.

4. Terapia Física. Universidad Autónoma del Estado de México. Ciudad de México, México.

\section{Resumen}

Introducción: Los sensores inerciales o unidad de medición inercial (IMU) del inglés Inertial measurement unit, son pequeños dispositivos capaces de medir la aceleración lineal y la velocidad angular, siendo útiles en el área de la salud para la cuantificación y valoración objetiva del movimiento corporal humano. Objetivo: Analizar la información sobre el uso de sensores inerciales como una aproximación a los procesos de evaluación del movimiento corporal humano. Materiales y métodos: Se realizó búsqueda en bases de datos, empleando términos: sensores inerciales, salud, fisioterapia, acelerómetro, actividad física, movimiento y rehabilitación, y sus combinaciones. Como criterios de exclusión se tuvo: artículos exclusivos del campo de ingeniería con información no aplicable a fisioterapia. Resultados: Una IMU es compatible con aplicaciones (APP), con el objetivo de obtener datos de movimiento tridimensionales y como evaluación e intervención, o que permita cuantificar los resultados de la acción motora. Conclusiones: Las IMU tienen amplias posibilidades en áreas afines a la rehabilitación y otras referentes al entrenamiento y el área deportiva; por lo, cual es necesario estandarizar protocolos que permitan la medición de patrones motores que favorezcan los procesos de rehabilitación.

Palabras clave: Fisioterapia; sensores inerciales; unidad de medición inercial; rehabilitación; evaluación en salud; movimiento. (Fuente: DeCS, Bireme).

\section{Abstract}

Introduction: Inertial measurement units (IMU) are small devices capable of measuring linear acceleration and angular velocity. Therefore, they are useful in the health field for the quantification and objective assessment of the human body movement. Objective: To analyze information about the inertial sensors usage, as a way to approach to processes of evaluation of the human body movement. Materials and methods: A database search was performed, using the following terms: inertial sensors, health, physiotherapy, accelerometer physical activity, movement, rehabilitation and their multiple combinations. The exclusion crite ria were exclusive articles from the engineering field covering information not relevant for physical therapy. Results: IMUs are devices that are compatible with applications, which can obtain three-dimensional movement data. They can also be used for assessment and intervention to quantify results of motor action. Conclusions: IMUs may have wide applications in fields such as rehabilitation, training and sports. As a result, it is necessary to standardize protocols to measure motor patterns and facilitate rehabilitation processes.

Palabras clave: Physiotherapy; inertial sensors; inertial measurement unit; rehabilitation; health evaluation; movement. (Fuente: DeCS, Bireme).

\footnotetext{
*Autor de correspondencia

Lina María Montealegre Mesa

e-mail: lmontealegre@autonoma.edu.co
} 


\section{Introducción}

Históricamente la tecnología ha tenido avances puestos al servicio de la humanidad, brindando mayor eficiencia, eficacia y bienestar, lo que concede al hombre la posibilidad de mejorar su calidad de vida, de allí que, en el área de la salud los avances tecnológicos de las últimas décadas van direccionados a la utilización de equipos y herramientas que contribuyan al trabajo de los profesionales de esta área, tanto en procesos de descubrimiento de nuevas, alternativas como para el abordaje de diversas condiciones de salud y de técnicas de evaluación e intervención de $\operatorname{vanguardia}^{(1)}$.

En este sentido, los sensores inerciales también denominados unidad de medición inercial o IMU (inertial measurement unit), son dispositivos compuestos por una masa, un par de resortes y un capacitor micro-maquinado, impregnados en un circuito monolítico, que permiten calcular la aceleración lineal y velocidad angular de los movimientos en tres ejes preestablecidos ( $\mathrm{x}, \mathrm{y}, \mathrm{z}) \mathrm{de}$ manera precisa y confiable ${ }^{(2,3)}$; lo anterior captando la información brindada en la ejecución de movimientos por medio de un acelerómetro, el cual logra calcular la velocidad en relación con la ejecución del movimiento, traduciendo esta información a aceleración; y los cambios posicionales que se encargan de brindar información a los giroscopios, ofreciendo así la posibilidad de medir el posicionamiento de una estructura corporal en relación con un punto fijo ${ }^{(4,5)}$.

Su auge comenzó el siglo pasado en la década de los 20 's donde eran caracterizados por ser de gran tamaño, pesados y de un alto costo, a partir de allí y con el paso del tiempo fueron evolucionando rápidamente hasta el punto de ser mucho más pequeños, fáciles de utilizar y portar(2). Estos sensores pueden procesar hasta tres ejes de movimiento de manera simultánea (frontal, coronal y transverso), con voltajes de alimentación y baterías pequeñas, los cuales permiten activarlos con rangos de medición y resolución para aplicaciones en el examen del movimiento humano(3), tales como los patrones de movimiento, velocidad en la ejecución de tareas específicas o en el desempeño de un gesto deportivo.

Las IMU se transforman en una herramienta novedosa en desarrollos tecnológicos, captura y análisis de movimiento(6) para su aplicación en diferentes disciplinas y en el caso particular de interés del presente artículo, en procesos de evaluación del movimiento corporal humano en fisioterapia, donde contribuyen al trabajo en ambientes no controlados, permitiendo, un análisis más efectivo e independiente de rangos de captura, velocidad de fotogramas o distancia de la cámara; facilitando así, que los movimientos se ejecuten con más libertad y armonía(7). Por lo anterior, el objetivo de esta revisión de literatura fue analizar la información sobre el uso de sensores inerciales como una aproximación a los procesos de evaluación del movimiento corporal humano.

\section{Materiales y métodos}

El presente artículo es un resultado de una revisión de literatura de tipo descriptivo, en la cual se realizó una búsqueda en bases de datos como Scopus, Research Gate, Science Direct, Dialnet, PubMed, Plos One, Scielo, Doaj y Google Scholar, empleando como términos: sensores inerciales, salud, fisioterapia, acelerómetro, actividad física, movimiento $\mathrm{y}$ rehabilitación, con las siguientes combinaciones: sensores inerciales $\mathrm{y}$ salud; sensor inercial $\mathrm{y}$ fisioterapia; sensores inerciales y movimiento; acelerómetro, salud y actividad física; acelerometría, actividad física, y rehabilitación; y términos en inglés: inertial sensors, movement, motion, accelerometer, mobility y activity y se desarrollaron las combinaciones: inertial sensors, motion and movement; sensors inercials and postural swing, inertial sensors and movement; y accelerometer, mobility and activity. De igual manera, se hizo uso de páginas web de entidades oficiales con la finalidad de extraer conceptos clave.

Los principales criterios de inclusión fueron: estudios relacionados con áreas osteomusculares, neurológicas, ergonómicas de la fisioterapia, así como sus interrelaciones con los análisis de movimiento corporal humano comprendidos entre los años 2010 y 2020; se excluyeron artículos exclusivos del campo de ingeniería con información no aplicable a fisioterapia. Posteriormente se registró la información de cada uno de los artículos en fichas de análisis bibliográficas, con el fin de determinar su aporte al objetivo de la revisión. Se consideraron artículos originales y completos para este fin. 


\section{Resultados}

En la Tabla 1 se muestran las aplicaciones de los sensores inerciales en el área de la salud. En este proceso se descargó un total de 103 registros, de los cuales se emplearon 53 artículos.

Las IMU son herramientas muy precisas en el proceso de medición de variables, que brindan a 1 fisioterapeuta, la posibilidad de trabajar interdisciplinariamente con profesionales de otras áreas como la ingeniería, con la finalidad de diseñar herramientas como hardware y software empleables en los procesos de rehabilitación funcional de personas, cuyas condiciones de salud se encuentran con una restricción en la ejecución de los patrones motrices; a través de sistemas que faciliten al portador una retroalimentación instantánea en los momentos de ejecución de patrones poco funcionales y que generen alto gasto energético, buscando por medio de un estímulo sensorial, hacer consciente al usuario de la necesidad de modificar la forma de realizar el patrón y la acción motriz.

Tabla 1. Resumen de usos y aplicaciones de las unidades de medición inercial o IMU en fisioterapia

\begin{tabular}{ll}
\hline \multicolumn{1}{c}{ Autores/ Año } & \multicolumn{3}{c}{ Usos y aplicaciones de los IMU'S en fisioterapia } \\
\hline Martínez, et al., 2013(2) & $\begin{array}{l}\text { Evaluar el balanceo postural y la factibilidad del uso de sensores inerciales para la } \\
\text { detección de movimientos posturales anticipatorios relacionados con } \\
\text { enfermedades neurodegenerativas. }\end{array}$ \\
\hline
\end{tabular}

López, et al., $2017^{(4)}$ Evaluar la viabilidad del uso de sensores inerciales y determinar diferencias entre grupos de personas en lo referente al movimiento corporal humano

Lee, et al., 2010(9)

Bötzel, et al., 2018(10) Evaluar la variables bajo el agua(9) de la marcha ${ }^{(10)(11)}$ en pruebas como la caminata

$\mathrm{Li}$, et al., 2018(11) de 6 minutos ${ }^{(12)}$ y en artroplastias de rodilla y cadera ${ }^{(13)(14) .}$

Proessl, et al., 2018 (12)

Tereso A., et al., 2015 (13)

Ferreira CVL., et al., 2018(14)

Tereso, et al., $2015^{(13)}$

Ruiz-Olaya, et al., 2017(15)

Cuantificar las diferencias en la cinemática(15)(16), la variabilidad del movimiento

Burns, et al., 2018 (16)

Burboa J., et al., 2018(17)

Cuesta y Williams 2011(18) de las sentadillas(13), cambios posturales durante ejercicios de resistencia.

Monroy EB., et al., 2020(19)

Leirós, et al., 2015(20)

Estudiar la cinemática de la manipulación vertebral y el comportamiento angular antes y después de una carga física $(17,18)$

Mantilla, 2017(21) Elaborar un protocolo de ubicación de sensores ${ }^{(18)}$ y acelerómetros(18) con el fin de prevenir úlceras por presión.

Leardini, et al., 2014(22)

Gómez, et al., 2018 (23)

Yasuma, et al., 2020(24)

Kim, et al., 2020(25)

Wilches, et al., 2007(26)

Beange, et al., 2019(27)

Ganesan, et al., $2015^{(28)}$

Wang, et al., 2017(29)

Fantozzi, et al., 2015(30)

Johnston, et al., 2019(31)

$\mathrm{Li}$, et al., 2020(32)

Liu, et al., 2018(33)

Caliandro, et al., 2019(34)

Gerber, et al., 2019 (35)

Wolff, et al., 2020 (36)

Emplear los acelerómetros en rehabilitación funcional de personas con condiciones de salud especifica ${ }^{(21)}$

Oubre, et al., 2020 (37) Utilizar las unidades IMU en rehabilitación física como forma de retroalimentación a los usuarios ${ }^{(22-26)}$ o a dispositivos como exoesqueletos, que participan en procesos de rehabilitación funcional $(28,29,30)$

Clemens, et al., $2020^{(38)}$

Evaluar el control postural(31), rangos de movimiento articular y patrones de movimiento $(32,33)$

Facilitar los procesos de neuro-rehabilitación en usuarios con parálisis cerebral, ataxia y secuelas de enfermedad cerebro vascular (34-37)

Permitir detectar diferencias biomecánicas en personas con amputaciones transfemorales y transtibiales (38) 
Los sensores inerciales establecen medidas en los procesos de micro y macro ciclos de atletas amateurs o élite, cuantificando las condiciones de velocidad y aceleración de gestos técnicos específicos $(2,8)$, o incluso medir fases y ciclos de actividades como la carrera y la marcha ${ }^{(9)}$; brindando la posibilidad de usar estos valores como puntos de referencia en test pre y post ejecución de planes de entrenamiento. En ese sentido, los sensores inerciales son dispositivos que pueden emplearse en procesos de investigación en general.

\section{Discusión}

Tradicionalmente, en fisioterapia las evaluaciones de rangos de movilidad articular, se han basado en el examen a través del uso de goniómetros convencionales, que constan de dos ejes y un punto fijo, los cuales ofrecen valores de ángulos de movimiento en articulaciones particulares y en movimientos fisiológicos (flexión, extensión, abducción, aducción y rotaciones); para ello, también se encuentran los electro-goniómetros e inclinómetros, que dan mayor precisión en la medición del rango articular. Además facilitan el abordaje de aquellos usuarios que revisten alteraciones funcionales en la movilidad, como resistencias articulares, que al ser medidos no permiten una evaluación objetiva; dichos dispositivos facilitan el almacenamiento de información que puede ser registrada en una base de datos para una posterior interpretación, sumando cada registro y dando la posibilidad de una identificación del movimiento más eficaz y real, lo cual favorece el seguimiento a procesos de intervención de forma más objetiva y precisa que los goniómetros convencionales en diferentes condiciones de salud(39).

No obstante, a pesar de las ventajas mencionadas del electro-goniómetro, esta herramienta no cuenta con la capacidad de registrar información adicional, relacionada con la aceleración y velocidad en movimientos desarrollados en planos y ejes específicos(40), variables que sí son registrables a través de las IMU en posiciones estáticas, dinámicas y durante la ejecución de actividades funcionales; pudiendo dar cuenta al fisioterapeuta de la riqueza de cuantificar patrones motrices funcionales que usualmente no se miden. Lo anterior, fortalece el examen a través del empleo de test y medidas novedosas, tanto para el proceso de evaluación como de seguimiento a la intervención fisioterapéutica (41).
Se observó que las IMU pueden desempeñar roles en procesos de rehabilitación funcional, la cual es entendida como "estrategias que permiten a los usuarios con problemas de salud mejorar, optimizar y mantener una función práctica en sus interacciones con el medio"(26) y de manera específica, en el examen de variables de medición que se pudieron estandarizar con los sensores inerciales, estos fueron la aceleración, angulación y cambios posicionales de la persona durante la ejecución de patrones de movimiento o actividades funcionales ${ }^{(7,31-33)}$.

A través de la historia los sensores inerciales gracias a su fiabilidad ${ }^{(15,42)}$ y a su poca desviación estándar al momento de arrojar los resultados, trascendieron del mundo de la ingeniería para ser empleados en otras áreas(43). En el campo de la fisioterapia y afines, su papel radicó en la interpretación y cuantificación del movimiento corporal humano(21) para ser analizados por medio de diferentes protocolos, donde se utilizan puntos anatómicos específicos para su colocación y posterior ejecución del movimiento(16,44).

Los IMU han apoyado diversos procesos evaluativos, permitiendo analizar el control postural(31), estabilidad de la columna vertebral(27), rangos de movimiento articular(7) en miembros superiores ${ }^{(45)} \mathrm{e}$ inferiores y la ejecución de patrones de movimiento como, realizar una estocada, correr(32,33), ascender y descender escaleras, marchar sobre superficie inclinada, declinada y nivelada(46), y en comparación con softwares de análisis de movimiento optoelectrónicos mostraron una buena correlación, resaltando su precisión y bajo costo $(34,7,14,33,46)$ lo cual perfila a estos dispositivos como una alternativa efectiva.

También se ha descrito la utilidad de los IMU en la evaluación de tareas funcionales(47) en poblaciones con alteraciones o síntomas específicos como dolor lumbar, permitiendo determinar la evolución, por medio de evaluaciones pre y post intervención fisioterapéutica(48), y clasificarlos de acuerdo a los datos cinemáticos obtenidos de los sensores(49), brindando ventajas en cuanto a la precisión y la calidad de la atención.

Por otra parte, estos dispositivos han sido empleados para medir niveles de actividad física en grupos poblacionales específicos $(2,6,50)$. Burboa et al.,(17) en su estudio, llevaron a cabo una relación 
entre los efectos del entrenamiento con respecto a los cambios en la angulación articular de miembros inferiores, encontrando que sí existe relación entre la fatiga generada en una sesión y los cambios en la angulación articular, siendo evidente la fiabilidad de las IMU en estas actividades. De igual manera, se emplearon como herramientas para la realización de evaluación de movimientos en diferentes entornos(13), haciendo uso de ellos en gran medida, en estudios relacionados con los parámetros de la marcha, en la medición de variables como velocidad, posicionamiento de los miembros inferiores(10), parámetros anormales de la marcha(11) y estimación de la rotación del movimiento de los usuarios para ser recreados y analizados con mayor profundidad en softwares de análisis de movimiento(51).

Otro importante uso es en la evaluación de cambios presentes en artroplastia de rodilla, por medio de dos sensores inerciales, uno conectado al tobillo de la pierna intervenida quirúrgicamente para medir los parámetros espaciotemporales de la marcha, y el otro en el sacro, para medir la postura y los parámetros relacionados con el riesgo de caídas(10).

En la artroplastia de cadera, en la cual se aplicó el Harris Hip Score para la evaluación específica, se ubicó una IMU en cada espina ilíaca anterosuperior del usuario, posteriormente el individuo debía caminar durante 3 minutos sobre una banda sin fin nivelada, para cada paso, se extrajeron las características de las señales del giroscopio del eje Y: valor pico, valor absoluto medio, desviación estándar y rango; para cada característica, se calculó una relación de simetría como la relación entre el lado izquierdo y el derecho(11).

Con respecto estructuras específicas de la articulación de la rodilla y en relación con su complejo ligamentoso, las IMU fueron útiles en la medición del desplazamiento angular, rotacional externo, aceleración del segmento(24) e índice de simetría de movimientos de las extremidades inferiores durante la prueba del escalón, estos se aplicaron en personas sanas y atletas con lesión de ligamentos de la rodilla, proporcionando un análisis cuantitativo para detectar diferencias cinemáticas después de una lesión de estas estructuras(25). Además, combinados con plataformas de fuerza, permitieron determinar alteración en la distribución de cargas de la rodilla y su relación con la velocidad angular de esta misma articulación, posterior a una reconstrucción de ligamento cruzado anterior(52); los resultados de estos estudios indicaron que estos dispositivos se perfilan como una herramienta complementaria para el cuerpo médico, en pro de facilitar la toma de decisiones con respecto al retorno del deportista, después de la lesión de los ligamentos de la rodilla(25).

En personas que se encontraban durante periodos prolongados en cama, se ubicaron sensores inerciales en las prendas de vestir como un medio para cuantificar el posicionamiento del usuario, y en combinación con sensores de presión, indicaron el momento en el cual se requerían cambios posicionales para prevenir así úlceras por presión(19).

Las IMU se han convertido en herramientas de apoyo en el área de la neuro-rehabilitación, específicamente en usuarios con ataxia cerebelosa para calcular la aceleración, el ritmo y el coeficiente de variación de la longitud del paso(34). Por otra parte, en usuarios con parálisis cerebral evaluaron el desempeño de la caminata diaria ${ }^{(35)}$ y el movimiento del miembro superior en tres velocidades distintas para determinar asimetrías en sus movimientos(36).

En pacientes con secuelas de accidente cerebrovascular, se midieron parámetros de movimiento como aceleración y cambios angulares en sus extremidades espásticas(37), demostrando la importancia del uso de la tecnología portátil(53) para determinar avances en los procesos de rehabilitación(7).

En otros estudios, como en la investigación realizada por Proessl et al.(12), las unidades IMU se emplearon en pruebas específicas como en la medición de variables de los patrones motores, ubicando seis sensores inerciales inalámbricos (APDM Opal) (en el esternón, en cada muñeca, espalda baja, a nivel de L5 y dorso de cada pie), a 20 adultos jóvenes neurológicamente sanos para realizar el test de marcha de 6 minutos, se midió la cadencia (pasos/min), velocidad de la marcha (m/s), zancada, longitud (m), duración (s) del paso y duración (s) de zancada $^{(7)}$.

En otro estudio(2), se hizo comparación entre una plataforma de fuerza y sensores inerciales para la identificación de movimientos posturales anticipatorios, lo cual demostró que es posible detectar y evaluar estos movimientos usando sólo 
sensores inerciales con una fiabilidad similar a la que brindan las plataformas de fuerza.

Por otro lado, Cuesta-Vargas y William(50), pretendieron determinar la capacidad de los sensores inerciales para el análisis de la cinemática de la manipulación vertebral cervical, fijando un sensor inercial a nivel del hueso frontal de la cabeza, analizaron manipulaciones cervicales a nivel de C56 , dando como resultado que los sensores inerciales son idóneos y presentan capacidad para dicha actividad.

En usuarios amputados se ha implementado el uso de IMU con el fin de detectar diferencias biomecánicas entre los segmentos indemnes y los protésicos, siendo empleados también, en la elaboración de prótesis de miembro superior para obtener datos que facilitan el monitoreo del proceso rehabilitatorio del usuario(38).

Asimismo, otros estudios describen la utilización de estos dispositivos para la creación de exoesqueletos, ubicándolos en la parte anterior de la muñeca para determinar la posición del exoesqueleto, y en conjunto con un sensor de electromiografía (EMG) identificar el ángulo de la flexión del antebrazo, para retroalimentar el sistema, lo cual permitió, la secuencia de trabajo y el movimiento del brazo del dispositivo(19).

Además los IMU han sido vinculados a sistemas de monitoreo inalámbrico en 3D para personas en rehabilitación física(17), sistemas de rehabilitación de tobillo en los cual se brinda una interfaz física basada en unidad IMU para medir la actitud del pie y una interfaz gráfica del usuario que actúa como feedback visual al realizar movimientos de pie, permitiendo la rehabilitación en el hogar involucrando al usuario en una terapia activa, por un precio asequible(44).

En la revisión de Wang et al.(29), cuyo objetivo fue inventariar y clasificar los sistemas portátiles interactivos para el monitoreo de movimientos y posturas con exoesqueletos(33) durante la rehabilitación de la parte superior del cuerpo, los resultados indicaron que el acelerómetro y el IMU, son la tecnología más utilizada dentro de los sistemas de retroalimentación para monitorear y proporcionar información sobre el rendimiento y el rango de movimiento durante la rehabilitación funcional de este segmento corporal(21).
Lo anterior concuerda con el estudio de Alizadegan y Behzadipour(52), en el que describen las IMU como herramienta de feedback efectiva en procesos de rehabilitación de estructuras como el hombro, o la muñeca posterior a una fractura distal de radio(45); combinado con aprendizaje supervisado, favorece el proceso, la participación e independencia del usuario(53). De igual manera, destaca la IMU gracias a que posee acelerómetro, magnetómetro y giroscopio, lo que le permite tener una funcionalidad al momento de ser utilizado en procesos de rehabilitación.

Todo esto, ha reflejado la importancia que tiene la utilización de sensores inerciales en fisioterapia, demostrando la amplia gama de funciones que se les pueden dar y la confiabilidad que brindan en diferentes procesos, específicamente en la evaluación del movimiento corporal humano(6) donde contribuyen a minimizar los errores, agilizar los procesos de evaluación(7) y permitir procesos de rehabilitación más precisos y específicos(33). Sin embargo, es necesario desarrollar estudios con muestras significativas que sustenten el uso de estos dispositivos en procesos rehabilitatorios(31,41).

\section{Conclusiones}

Aunque las unidades de medición inercial o IMU han sido empleadas en la fisioterapia, aún tienen mucho campo por conquistar, no sólo en áreas afines a la medicina sino también en campos referentes al entrenamiento y la rehabilitación deportiva. Las IMU se perfilan como una alternativa de gran utilidad en procesos de rehabilitación funcional planeados y ejecutados por fisioterapeutas $y$ terapeutas ocupacionales.

Es necesario recalcar la necesidad de generalizar protocolos de ubicación anatómica la IMU, de tal manera que permitan un óptimo rendimiento y la estandarización de medidas universales en cuanto al cálculo de la velocidad angular y la aceleración lineal, que sean útiles en el monitoreo de actividades básicas cotidianas y actividades de la vida diaria de las personas.

\section{Agradecimientos}

A la Universidad Autónoma de Manizales, a su laboratorio de análisis de movimiento y a los integrantes del semillero Tecnología Aplicada $\mathrm{Al}$ Movimiento desde la Ingeniería y la Fisioterapia- 
TAMIF, por facilitar los espacios académicos que permitieron esta revisión.

\section{Conflicto de intereses}

Los autores declaramos no poseer ningún conflicto de interés respecto de la información presentada en este texto académico.

\section{Referencias}

1. Betances Reinoso FA, López Montes T, Rodríguez Ontiveros VM, Chiesa Estomba C. Análisis de la marcha y el equilibrio mediante el uso de sensores inerciales: estudio prospectivo, longitudinal, no aleatorio. cysa [Internet]. 3 de marzo de 2020 [citado 4 de marzo de 2020];4(1):11-6. Disponible en: https://revistas.intec.edu.do/index.php/cisa/article/view/ 1671

2. Martínez Méndez R, Portillo Rodríguez 0, Romero Huertas M, Vilchis González A. Uso de sensores inerciales en la medición y evaluación de movimiento humano para aplicaciones en la salud. Ideas en ciencia. 2012;37.

3. Sanz J, Arbeláez Salazar O. Determinación de la aceleración, velocidad y desplazamiento utilizando acelerómetros micromaquinados. Scientia Et Technica.2004;1(24). https://revistas.utp.edu.co/index.php/revistaciencia/articl e/view/7291

4. López-Nava IH, Arnrich B, Muñoz-Mendez A, Güneysu A. Variability Analysis of Therapeutic Movements using Wearable Inertial Sensors. J Med Syst. 2017;41(7). https://doi.org/10.1007/s10916-016-0645-8.

5. Gleadhill S, Bruce J, James D. The development and validation of using inertial sensors to monitor postural change in resistance exercise. J Biomech [Internet]. 2016;49(7):1259-63. Available from: http://dx.doi.org/10.1016/j.jbiomech.2016.03.012

6. Santos TMO, Barroso MFS, Ricco RA, Nepomuceno EG, Alvarenga ÉLFC, Penoni ÁCO, et al. A low-cost wireless system of inertial sensors to postural analysis during human movement. Meas J Int Meas Confed [Internet]. 2019; 148:106933. Available from: https://doi.org/10.1016/j.measurement.2019.106933

7. Ong ZC, Seet YC, Khoo SY, Noroozi S. Development of an economic wireless human motion analysis device for quantitative assessment of human body joint. Meas J Int Meas Confed [Internet]. 2018;115(August 2017):306-15. Available from: http://dx.doi.org/10.1016/j.measurement.2017.10.056

8. Aguilar Cordero MJ, López AMS, Barrilao RG. Descripción del acelerómetro como método para valorar la actividad física en los diferentes periodos de la vida; revisión sistemática. Nutr. Hosp. 2014;29(6):1250-61. Available from: http://dx.doi.org/10.3305/nh.2014.29.6.7410

9. Lee JB, Mellifont RB, Burkett BJ. The use of a single inertial sensor to identify stride, step, and stance durations of running gait. J Sci Med Sport. 2010 Mar;13(2):270-3. doi: 10.1016/j.jsams.2009.01.005. Epub 2009 Jul 1. PMID: 19574098.

10. Bötzel K, Olivares A, Cunha JP, Górriz Sáez JM, Weiss R, Plate A. Quantification of gait parameters with inertial sensors and inverse kinematics. J Biomech. 2018;72:207-14.

11. Li G, Liu T, Yi J. Wearable Sensor System for Detecting Gait Parameters of Abnormal Gaits: A Feasibility Study. IEEE Sens J. 2018;18(10):4234-41.
12. Proessl F, Swanson CW, Rudroff T, Fling BW, Tracy BL. Good agreement between smart device and inertial sensor-based gait parameters during a 6-min walk. Gait \& Posture. 2018;64(March):63-7. Available from: https://doi.org/10.1016/j.gaitpost.2018.05.030

13. Tereso A, Martins MM, Santos CP. Evaluation of gait performance of knee osteoarthritis patients after total knee arthroplasty with different assistive devices. Research on Biomedical Engineering. 2015;31(3):208-17. Available from: http://dx.doi.org/10.1590/2446-4740.0729

14. Ferreira CVL, Rabelo AG, Vieira MF, Pereira AA, Andrade A de 0 . Gait variability and symmetry assessment with inertial sensors for quantitative discrimination of Trendelenburg sign in total hip arthroplasty patients: a pilot study based on convenience sampling. Research on Biomedical Engineering. 2018;34(1):65-72. Available from: https://doi.org/10.1590/2446-4740.07017

15. Ruiz-Olaya AF, Callejas-Cuervo $\mathrm{M}$, Lara-Herrera $\mathrm{CN}$. Wearable low-cost inertial sensor-based electrogoniometer for measuring joint range of motion. DYNA. 2017;84(201):180-185. Available from: https://dx.doi.org/10.15446/dyna.v84n201.59054

16. Burns D, Leung N, Hardisty M, Whyne C, Henry P, McLachlin $S$. Shoulder physiotherapy exercise recognition: machine learning the inertial signals from a smartwatch. Physiol. Meas. 2018;39(7). Available from: https://doi.org/10.1088/1361-6579/aacfd9

17. Burboa J, Bahamonde $M$, Inostroza $M$, Lillo $P$, Barahona $M$, Palet $M$, et al. Efecto de un entrenamiento deportivo en el compartimiento angular de la extremidad inferior en niños futbolistas de entre 11 y 12 años. Acta ortop. Mex. 2017;31(3):128-33. Available from: http://www.scielo.org.mx/scielo.php?script=sci_arttext\&pi $\mathrm{d}=$ S2306-41022017000300128\&lng=es.

18. Monroy EB, Rodriguez AP, Estevez ME, Quero JM. Fuzzy Monitoring of In-bed Postural Changes for the Prevention of Pressure Ulcers using Inertial Sensors Attached to Clothing. J Biomed Inform [Internet]. 2020;107:103476. Available from: https://doi.org/10.1016/j.jbi.2020.103476

19. Mantilla A. Usos de los acelerómetros en fisioterapia: una revisión de la literatura uses of accelerometers in physiotherapy: a review of the literature. Rev.Ib.CC. Act. Fís. Dep. 2017;6(2):38-45.

20. Leardini A, Lullini G, Giannini S, Berti L, Ortolani M, Caravaggi P. Validation of the angular measurements of a new inertial-measurement-unit based rehabilitation system: comparison with state-of-the-art gait analysis. J Neuroeng Rehabil. 2014 Sep 11;11(136). doi: 10.1186/1743-0003-11-136. PMID: 25212257; PMCID: PMC4169865.

21. Gómez Espinosa A, Espinosa-Castillo N, Valdes-Aguirre B. Foot-Mounted Inertial Measurement Units-Based Device for Ankle Rehabilitation. Appl. Sci. 2018;10(8):2032. Doi:10.3390/app8112032.

22. Yasuma S, Nozaki M, Murase A, Kobayashi M, Kawanishi $Y$, Fukushima H, Takenaga T, Yoshida M, Kuroyanagi G, Kawaguchi Y, Nagaya Y, Murakami H. Anterolateral ligament reconstruction as an augmented procedure for doublebundle anterior cruciate ligament reconstruction restores rotational stability: Quantitative evaluation of the pivot shift test using an inertial sensor. Knee. 2020 Mar;27(2):397405. doi: 10.1016/j.knee.2020.02.015. Epub 2020 Mar 13. PMID: 32178972. 
23. Kim KJ, Gailey R, Agrawal V, Gaunaurd I, Feigenbaum L, Bennett $\mathrm{C}$, et al. Quantification of agility testing with inertial sensors after a knee injury. Med Sci Sports Exerc. 2020;52(1):244-51. doi: 10.1249/MSS.0000000000002090

24. Wilches EC, López ME, Arango GP. Rehabilitación funcional del paciente neurológico en la UCI. Guía Neurológica 6 - La unidad de cuidado intensivo 2004. Capítulo 7 p: 119-142.

25. Beange KHE, Chan ADC, Beaudette SM, Graham RB. Concurrent validity of a wearable IMU for objective assessments of functional movement quality and control of the lumbar spine.J Biomech. 2019;97:109356. doi: 10.1016/j.jbiomech.2019.109356

26. Ganesan Y, Gobee S, Durairajah V. Development of an Upper Limb Exoskeleton for Rehabilitation with Feedback from EMG and IMU Sensor. Procedia - Procedia Comput Sci [Internet]. 2015;76(Iris):53-9. Available from: http://dx.doi.org/10.1016/j.procs.2015.12.275

27. Wang Q, Markopoulos P, Yu B, Chen W, Timmermans A. Interactive wearable systems for upper body rehabilitation: a systematic review. J Neuroeng Rehabil. 2017 Mar 11;14(1):20. doi: 10.1186/s12984-017-0229-y. PMID: 28284228; PMCID: PMC5346195.

28. Johnston W, O’Reilly M, Argent R, Caulfield B. Reliability, Validity and Utility of Inertial Sensor Systems for Postural Control Assessment in Sport Science and Medicine Applications: A Systematic Review [Internet]. Sports Medicine. Springer International Publishing. 2019;49:783818 p. Available from: https://doi.org/10.1007/s40279019-01095-9

29. Li R, Jumet B, Ren H, Song W, Tse ZTH. An inertial measurement unit tracking system for body movement in comparison with optical tracking. Proc Inst Mech Eng H. $2020 \quad$ Jul;234(7):728-737. doi: 10.1177/0954411920921695. Epub 2020 May 18. PMID: 32419605.

30. Liu K, Yan J, Liu Y, Ye M. Noninvasive Estimation of Joint Moments with Inertial Sensor System for Analysis of STS Rehabilitation Training. J Healthc Eng. 2018 Feb 11; 2018:115. doi: 10.1155/2018/6570617. PMID: 29610656; PMCID: PMC5828652.

31. Caliandro P, Conte C, Iacovelli C, Tatarelli A, Castiglia SF, Reale G, et al. Exploring risk of falls and dynamic unbalance in cerebellar ataxia by inertial sensor assessment. Sensors (Switzerland). 2019;19(24):1-9. Available from: https://doi.org/10.3390/s19245571

32. Gerber CN, Carcreff L, Paraschiv-Ionescu A, Armand S, Newman CJ. Reliability of single-day walking performance and physical activity measures using inertial sensors in children with cerebral palsy. Ann Phys Rehabil Med. 2019;(2018):2-7. Available from: https://doi.org/10.1016/j.rehab.2019.02.003

33. Wolff A, Sama A, Lenhoff M, Daluiski A. The use of wearable inertial sensors effectively quantify arm asymmetry during gait in children with unilateral spastic cerebral palsy. J Hand Ther [Internet]. 2020;2-3. Available from: https://doi.org/10.1016/j.jht.2020.03.026

34. Oubre B, Daneault JF, Jung HT, Whritenour K, Miranda JGV, Park J, et al. Estimating Upper-Limb Impairment Level in Stroke Survivors Using Wearable Inertial Sensors and a Minimally-Burdensome Motor Task. IEEE Transactions on Neural Systems and Rehabilitation Engineering. 2020;28(3):601-11. Available from: https://doi.org/10.1109/tnsre.2020.2966950
35. Clemens S, Kim KJ, Gailey R, Kirk-Sanchez N, Kristal A, Gaunaurd I. Inertial sensor-based measures of gait symmetry and repeatability in people with unilateral lower limb amputation. Clin Biomech [Internet]. 2020;72(December 2019):102-7. Available from: https://doi.org/10.1016/j.clinbiomech.2019.12.007

36. Montoya-Leal V, Pérez VZ. Valoración cuantitativa para la reincorporación ocupacional. Revista Científica Salud Uninorte. 2016;32(2):319-36. Available from: http://dx.doi.org/10.14482/sun.32.2.8836

37. Carnaz L, Moriguchi CS, de Oliveira AB, Santiago PRP, Caurin GAP, Hansson GA, et al. A comparison between flexible electrogoniometers, inclinometers and three-dimensional video analysis system for recording neck movement. Med Eng Phys [Internet]. 2013;35(11):1629-37. Available from: http://dx.doi.org/10.1016/j.medengphy.2013.05.014

38. Gordt K, Gerhardy T, Najafi B, Schwenk M. Effects of Wearable Sensor-Based Balance and Gait Training on Balance, Gait, and Functional Performance in Healthy and Patient Populations: A Systematic Review and MetaAnalysis of Randomized Controlled Trials. Gerontology. 2017;64(1):74-89.

39. Saber-Sheikh K, Bryant EC, Glazzard C, Hamel A, Lee RYW. Feasibility of using inertial sensors to assess human movement. Man Ther [Internet]. 2010;15(1):122-5. Available http://dx.doi.org/10.1016/j.math.2009.05.009

40. Fantozzi S, Giovanardi A, Borra D, Gatta G. Gait Kinematic Analysis in Water Using Wearable Inertial Magnetic Sensors. PLoS One. 2015 Sep 14;10(9):e0138105. doi: 10.1371/journal.pone.0138105. PMID: 26368131; PMCID: PMC4569370.

41. Leirós-Rodríguez R, Arce ME, Souto-Gestal A, García-Soidán JL. Identificación de puntos de referencia anatómicos para la valoración del equilibrio mediante dispositivos cinemáticos. Fisioterapia [Internet]. 2015;37(5):223-9. Available from: http://dx.doi.org/10.1016/j.ft.2014.10.006

42. Zucchi B, Mangone M, Agostini F, Paoloni M, Petriello L, Bernetti A, et al. Movement Analysis with Inertial Measurement Unit Sensor after Surgical Treatment for Distal Radius Fractures. Biores Open Access. 2020;9(1):151-61.

43. Mundt M, Thomsen W, David S, Dupré T, Bamer F, Potthast $\mathrm{W}$, et al. Assessment of the measurement accuracy of inertial sensors during different tasks of daily living. J Biomech [Internet]. 2019;84:81-6. Available from: https://doi.org/10.1016/j.jbiomech.2018.12.023

44. Tulipani L, Boocock MG, Lomond KV, El-Gohary M, Reid DA, Henry SM. Validation of an Inertial Sensor System for Physical Therapists to Quantify Movement Coordination During Functional Tasks. J Appl Biomech. 2018 Feb 1;34(1):23-30. doi: 10.1123/jab.2016-0139. Epub 2018 Feb 7. PMID: 28787248.

45. Celletti C, Mollica R, Ferrario C, Galli M, Camerota F. Functional evaluation using inertial measurement of back school therapy in lower back pain. Sensors (Switzerland). 2020;20(2):1-7.

46. Abdollahi M, Ashouri S, Abedi M, Azadeh-Fard N, Parnianpour M, Khalaf K, Rashedi E. Using a Motion Sensor to Categorize Nonspecific Low Back Pain Patients: A Machine Learning Approach. Sensors (Basel). 2020 Jun 26;20(12):3600. doi: 10.3390/s20123600. PMID: 32604794; PMCID: PMC7348921. 
47. Cuesta-Vargas AI, William JM. Estudio de la cinemática y fiabilidad inter e intraterapeutas de la manipulación vertebral cervical basada en sensores inerciales. Fisioterapia. 2011;33(1):25-30. Available from: https://doi.org/10.1016/j.ft.2010.12.005

48. Yang P, Xie L, Wang C, Lu S. Demo: IMU-Kinect: A Motion Sensor-based Gait Monitoring System for Intelligent Healthcare. UbiComp/ISWC 2019 - Adjun Proc 2019 ACM Int Jt Conf Pervasive Ubiquitous Comput Proc 2019 ACM Int Symp Wearable Comput. 2019;350-3. Available from: https://doi.org/10.1145/3341162.3343766

49. Sigward SM, Chan MSM, Lin PE. Characterizing knee loading asymmetry in individuals following anterior cruciate ligament reconstruction using inertial sensors. Gait Posture [Internet]. 2016;49:114-9. Available from: http://dx.doi.org/10.1016/j.gaitpost.2016.06.021

50. Kim JY, Park G, Lee SA, Nam Y. Analysis of machine Learning based assessment for elbow spasticity using inertial sensors. Sensors (Switzerland). 2020;20(6):1-15. Available from: https://doi.org/10.3390/s20061622

51. Gaetani F, de Fazio R, Zappatore GA, Visconti P. A prosthetic limb managed by sensors-based electronic system: Experimental results on amputees. Bull Electr Eng Informatics. 2020;9(2):514-24. Available from: https://doi.org/10.11591/eei.v9i2.2101

52. Alizadegan A, Behzadipour S. Shoulder and elbow joint angle estimation for upper limb rehabilitation tasks using low-cost inertial and optical sensors. J Mech Med Biol. 2017;17(2):1-20. Available from: https://doi.org/10.1142/S0219519417500312

53. Bavan L, Surmacz K, Beard D, Mellon S, Rees J. Adherence monitoring of rehabilitation exercise with inertial sensors: A clinical validation study. Gait Posture [Internet]. 2019;70(February):211-7. Available from: https://doi.org/10.1016/j.gaitpost.2019.03.008 